\title{
LIMITS OF CONFINEMENT ENHANCEMENT FOR STELLARATORS
}

\author{
F. Warmer, C.D. Beidler, A. Dinklage, Y. Turkin, R. Wolf \\ Max-Planck-Institut für Plasmaphysik, D-17491 Greifswald, Germany
}

\begin{abstract}
In fusion power plant studies a high confinement improvement with respect to empirical scalings is often assumed in order to design compact machines. In this work the limits of such a confinement enhancement is studied for helical-axis advanced stellarators (HELIAS).

As a first exercise, the well-established power balance approach is used to investigate the impact of confinement enhancement (in terms of the ISS04 renormalisation factor) on the required size of HELIAS power plants. It is found that both a lower (0.5) and an upper limit $(1.5-1.7)$ exists for which, respectively, ignition is no longer possible or further confinement enhancement irrelevant due to physics limits.

In the second part of the work a predictive neoclassical transport model is introduced and employed in order to determine a selfconsistent confinement time based on transport modelling. It is found that the confinement enhancement with respect to the ISS04 scaling decreases in comparison to $\mathrm{W} 7-\mathrm{X}$ as the device is scaled to reactor size dropping from $\sim 2.5$ to $1.2-1.3$. This behaviour is explained with underlying scaling relations and transport effects. The results from both models are consistent and important for future HELIAS systems studies.
\end{abstract}

Keywords: HELIAS, scaling laws, renormalisation factor, confinement enhancement

\section{INTRODUCTION}

The ultimate goal of fusion research is to demonstrate the feasibility of the economic production of electricity. To achieve this goal, extrapolation to burning plasma devices beyond the scope of current experiments is necessary. Such studies allow one to identify critical technology and research areas to assess necessary future steps, e.g. experiments, simulations and technology development, on the way to fusion power plants. In addition, the dimensions and design parameters of such devices can be estimated.

In the assessment of the required size of stellarator power plants, a critical parameter is the confinement enhancement with respect to empirical scaling laws. As stellarators are subject to high neoclassical transport, such enhancement of the confinement is assumed by postulating further optimisation of magnetic configurations.

From the variety of stellarator magnetic configurations, currently two major, promising concepts are explored in larger experiments. On the one hand, the heliotron-line is investigated with the Large Helical Device (LHD) in Japan where the magnetic field is created by continuous coils. On the other hand the helical-axis advanced stellarator-line (HELIAS) will be studied in the Wendelstein 7-X (W7-X) experiment in Germany employing a modular coil set with a 5-period symmetry.

Based on these concepts several reactor studies have been carried out in the past. For the heliotron-line these center

Email address: felix.warmer@ipp.mpg.de (F. Warmer) around the notion of the Force Free Helical Reactor (FFHR) (Ref. 1) while HELIAS power plant studies have considered four and five field period candidates, HSR4/18 and HSR5/22 (Refs. 2, 3) respectievely. The corresponding machine parameters are summarised in Tab. I]

In the references cited different assumptions on confinement enhancement over existing empirical scalings are made but have not been thoroughly checked for consistency. Therefore, in this work the limits of confinement enhancement are investigated (with focus on the helical-axis advanced stellarator line) and their impact on the design process of fusion power plants discussed. Being a complex topic, this work is here seperately disussed from the general HELIAS systems studies ${ }^{4,5}$ which apart from confinement properties take many other limitations into account such as neutron wall load, divertor exhaust, as well as engineering considerations. These studies are still ongoing and not subject of this work.

This work is organised as follows: in section II a 0-D global power balance model for a stellarator burning plasma device is derived using the approach of Ref. ${ }^{6}$ Before the model is employed, stellarator-specific physics constraints on the magnetic field strength $B_{t}$ and on the normalised plasma pressure $\langle\beta\rangle=1 / V \int \mathrm{d} V 2 \mu_{0} \sum n k T / B^{2}$ are discussed and the wellknown sensitivity on fusion power illustrated. The section is concluded with an analysis of the renormalisation factor, $f_{\text {ren }}$, (representing confinement enhancement / degradation depending on the magnetic field structure) and its impact on the device size required to achieve ignition for a 4- and 5-fieldperiod HELIAS. The section concludes with a direct extrapolation of W7- 


\begin{tabular}{llll} 
& W7-X & HSR4/18 & HSR5/22 \\
\hline Major radius $R_{0}[\mathrm{~m}]$ & 5.5 & 18 & 22 \\
Average minor radius $a[\mathrm{~m}]$ & 0.53 & 2.1 & 1.8 \\
Plasma Volume $V\left[\mathrm{~m}^{3}\right]$ & 30 & 1570 & 1407 \\
Average magnetic field on axis $B_{t}[\mathrm{~T}]$ & 2.5 & 5.0 & 5.0 \\
Number of coils & 50 & 40 & 50 \\
Line averaged electron density $\bar{n}_{e}\left[10^{20} \mathrm{~m}^{-3}\right]$ & $<2.0$ & 2.6 & 2.12 \\
Central electron temperature $T_{0}[\mathrm{keV}]$ & $<5.0$ & 15 & 15 \\
Average plasma $\langle\beta\rangle[\%]$ & $<5.0$ & 4.2 & 4.2 \\
\hline
\end{tabular}

TABLE I: Main physics and engineering parameters for W7-X, HSR4/18 and HSR5/22.

$\mathrm{X}$ to power plant conditions under the constraints mentioned above.

Next, in section III, a predictive 1-D neoclassical transport model is introduced. This model allows the simulation of the transport in an up-scaled W7-X high-mirror configuration. The confinement times predicted by the model are compared against the empirical ISS04 scaling ${ }^{7}$ and the discrepancies found (in the sense of a confinement enhancement factor) are highlighted and explained. Finally the results from the 0-D and 1-D model are compared yielding consistent conclusions. The various results and implications of the work are discussed and summarised in section IV

\section{GLOBAL POWER BALANCE APPROACH}

In the following section, a basic power balance model is derived to identify the predominant parameters relevant to arrive at the conditions for plasma ignition. Such approaches are widely used with similar methodologies.

For further discussions in this paper, the energy confinement, being a relevant quantity in the model, was investigated and physical and technical constraints introduced. The energy confinement is quantified through the energy confinement time $\tau_{E}$ and empirical scaling laws give its relation to geometry, heating power, plasma density and magnetic field properties. For the most recent stellarator scaling ISS04 (Refs. 7, 8) this reads:

$$
\tau_{E}^{\mathrm{ISS} 04}=f_{\text {ren }} \cdot 0.134 a^{2.28} R^{0.64} P^{-0.61} \bar{n}_{e}^{0.54} B_{t}^{0.84} \epsilon_{2 / 3}^{0.41}
$$

where $a$ is the plasma minor radius in $\mathrm{m}, R$ the major radius in $\mathrm{m}, P$ the heating power in $\mathrm{MW}, \bar{n}_{e}$ the line-averaged electron density in $10^{19} \mathrm{~m}^{-3}, B_{t}$ the magnetic field strength on axis in $\mathrm{T}$, and $t_{2 / 3}$ the rotational transform at $2 / 3$ of the minor radius. The renormalisation factor, $f_{\text {ren }}$, can serve the function of a confinement enhancement / degradation factor similar to the $H$-factor used in tokamaks but, for stellarators, $f_{r e n}$ also reflects the complex structure of stellarator magnetic fields and is therefore dependent on the magnetic configuration.

Employing the definition of confinement time $\tau_{E}$ and taking the ISS04 scaling, the power leaving the plasma through transport processes becomes $P_{\text {loss }}=W / \tau_{E}$ where $W$ is the plasma energy. Assuming on the one hand toroidal geometry and on the other hand that density and temperature only depend on the minor radius $r$ (in analogon to nested flux surfaces) the plasma energy may be described by

$$
W=\frac{3}{2}(2 \pi a)^{2} R_{0} \int_{0}^{1} \mathrm{~d} \rho \rho \sum_{j} n_{j}(\rho) T_{j}(\rho)
$$

where $\rho=r / a$ is the normalised minor radius and $n_{j}, T_{j}$ are the local density and temperature of the particle species $j=e$, $i$ (electrons or ions). Neglecting for the moment profile effects, the local plasma pressure $p=\sum_{j} n_{j} k T_{j}$ can be replaced by the volume-averaged plasma beta $\langle\beta\rangle \sim p / B_{t}^{2}$. The profile dependencies are collected in a constant $c_{1}$ such that the plasma energy becomes the simple expression

$$
W=c_{1} \frac{\langle\beta\rangle B_{t}^{2} R_{0}^{3}}{A^{2}}
$$

where $A$ is the aspect ratio $A=R_{0} / a$. The profile parameter $c_{1}$ was chosen such that it agrees with the results from the HSR4/18 and HSR5/22 reactor studies.

The fusion power produced by such a device, under the same assumptions, can be expressed as

$$
P_{f u s}=E(2 \pi a)^{2} R_{0} \int_{0}^{1} \mathrm{~d} \rho \rho n_{D}(\rho) n_{T}(\rho)\langle\sigma v\rangle(\rho)
$$

with $E=17.6 \mathrm{MeV}$ being the energy released by one D-T fusion reaction. With $n_{D}=n_{T}=n_{e} / 2$ and $T_{D}=T_{T}=T_{e}$ it is possible to approximate the rate coefficient of the D-T reaction by $\langle\sigma v\rangle \sim T^{2}$ in the relevant reactor temperature regime $10 \mathrm{keV}<T<20 \mathrm{keV}$. The fusion power then becomes $P_{f u s}=E(\pi a)^{2} R_{0} \int_{0}^{1} \mathrm{~d} \rho \rho n_{e}^{2}(\rho) T^{2}(\rho)$. Combining again density and temperature as the pressure $p$ and replacing this by the volume-averaged $\langle\beta\rangle$ the simple relation

$$
P_{\text {fus }}=c_{2} \frac{\langle\beta\rangle^{2} B_{t}^{4} R_{0}^{3}}{A^{2}}
$$

emerges with an additional profile parameter $c_{2}$ similarly chosen to agree with HSR4/18 and HSR5/22. The values are summarised in Tab. III

In addition to the fusion power and the plasma transport losses it is necessary to characterise the power balance of such a fusion reactor. The flow chart of the power balance treated here is illustrated in Fig. 11. As the aim of this work was the investigation of global effects only, the power flow is not broken down to smaller scales. The useable thermal energy in this 


\begin{tabular}{lll} 
Configuration & $c_{1}$ & $c_{2}$ \\
\hline HSR4/18 & 0.1175 & 0.004276 \\
HSR5/22 & 0.1169 & 0.004215 \\
\hline
\end{tabular}

TABLE II: Values of the profile parameters $c_{1}$ and $c_{2}$ of the global power balance model determined to comply with the results of the HSR4/18 and HSR5/22 reactor studies.

work is defined as the total fusion power modified with a factor $M$. This additional factor takes into account the additional energy generated by nuclear reactions in the blanket / divertor. Thus, the thermal power $P_{t h}$ produced in this model reads

$$
P_{\text {th }}=M \cdot P_{\text {fus }}+P_{\text {Heat }}+\eta_{\text {BoP }} P_{\text {BoP }} .
$$

The integrated cooling system, with a thermal conversion efficiency $\eta_{t h}$, converts the thermal power to the gross electrical power $P_{e l}=\eta_{t h} P_{t h}$. A fraction of this gross electrical power must be recirculated within the power plant itself to operate the various subsystems. In this study this power is referred to as the auxiliary power $P_{A U X}=f_{r e c} P_{e l}$. The remaining net electrical power can than be provided to the grid.

The auxiliary power in turn consists predominantly of two components. One component is the power required for the balance of plant systems, $P_{B o P}$, mainly driven by the required pumping power $P_{B o P} \cong P_{\text {pump }}$. The other component is the additional heating power, $P_{\text {Heat }}$, needed to compensate the plasma transport and radiation losses in excess of alpha particle heating: $P_{\text {Heat }}=P_{\text {loss }}+P_{\text {rad }}-P_{\alpha}$. Here, $P_{\text {rad }}$ is the power loss by radiation that in a stellarator reactor mainly comes from bremsstrahlung, as synchrotron and line radiation are negligible in comparison, as long as impurities are not introduced on purpose to increase core radiation. With the heating system efficiency $\eta_{\text {heat }}$ the power required to operate the system is $P_{\text {Heat }, \text { Gross }}=P_{\text {Heat }} / \eta_{\text {heat }}$.

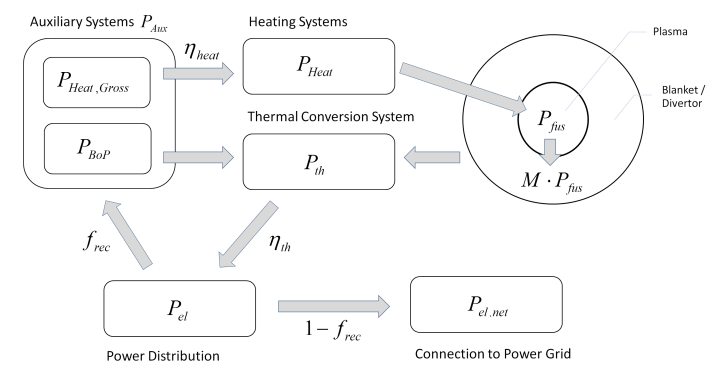

Fig. 1: Flow chart for the basic power balance model of stellarator burning plasma devices.

\section{II.A Magnetic Field Strength and Plasma Beta}

The strength of the magnetic field has a significant influence on the global confinement as can be seen in the empirical confinement time ISS04 $\left(\tau_{E} \sim B_{t}^{0.84}\right)$, introduced at the beginning of this section. Moreover, the substitution of the plasma pressure $p \propto\langle\beta\rangle B_{t}^{2}$ reveals a sharp dependence of the plasma energy and of the fusion power on the magnetic field strength $B_{t}$ and $\langle\beta\rangle$ (equations (3) and (4)). Again, as is well known, an increase of both parameters is beneficial for the global performance and the constraints for both $B_{t}$ and $\langle\beta\rangle$ are therefore highlighted.

\section{II.A.1. Physics and Engineering Constraints}

For $B_{t}$ it was shown in ${ }^{9}$ for a 4 -periodic HELIAS that $4.4 \mathrm{~T}$ magnetic field can be achieved on axis using existing NbTi technology with conventional helium cooling at $4.2 \mathrm{~K}$. The field in this case could be pushed higher up to $5 \mathrm{~T}$ if super-critical helium at $1.8 \mathrm{~K}$ is applied as indicated in. ${ }^{2}$ With the advancement of superconductor material development and the large scale use of $\mathrm{Nb}_{3} \mathrm{Sn}$ in ITER, it was concluded in ${ }^{10}$ that the $\mathrm{Nb}_{3} \mathrm{Sn}$ technology could be safely employed for stellarators allowing a magnetic field strength of up to $5.6 \mathrm{~T}$ on axis. However, the application of new materials is limited by availability, costs and the capability of complex 3D shaping. It was also shown in ${ }^{11}$ that the forces and stresses arising in 5-period HELIAS with $R_{0}=22 \mathrm{~m}$ and a magnet system achieving $5.6 \mathrm{~T}$ on axis are manageable.

While the achievable magnetic field is more an engineering issue the constraints of the achievable $\langle\beta\rangle$ are driven by physics considerations. A HELIAS may operate only within a certain 'window' of $\langle\beta\rangle$. That means both a minimum threshold and a maximum limit on the value of $\langle\beta\rangle$ exists. The lower $\langle\beta\rangle$ limit is related to the confinement of the fast $\alpha$-particles. In order to confine the collisionless $\alpha$ 's in a HELIAS configuration, so-called 'minimum- $B$ ' configurations are necessary. To achieve these configurations the diamagnetic effect of the plasma is necessary providing a deeper magnetic well and a faster poloidal $\nabla B$ drift beneficial for the fast particle confinement. The minimum- $B$ configurations are not achieved until the local $\beta$ is sufficiently large. As the before-mentioned effect gets stronger with increasing $\beta$, dependent on the profiles a certain minimal value of $\langle\beta\rangle$ must be reached. This value is dependent on the magnetic well in the specific vacuum configuration ${ }^{12}$ and for the HELIAS line lies typically around $\langle\beta\rangle=3-4 \%$.

The maximum $\langle\beta\rangle$ is anticipated to have a 'soft' limit coming from an increase of MHD instabilities gradually enhancing transport and decreasing confinement, but it is 'soft' in the sense that stellarators are observed to still operate above the ideal MHD ballooning limit and beyond the Mercier limit. ${ }^{13,14,15,16}$ Linear MHD stability considerations yield a beta limit of $4.5 \%$ in W7-X. But as already pointed out, stellarators are observed to operate above such limits reducing their credibility. In the end, $\langle\beta\rangle$ may be limited by equilibrium-considerations, namely the stochastisation of the magnetic field at increasing beta. At increasing $\langle\beta\rangle$ the magnetic field becomes stochastic at the edge causing a continuous destruction of flux surfaces such that this stochastic region expands thereby decreasing the plasma volume. This effect could ultimately limit beta to the range $5-6 \%$ as suggested by theoretical studies. ${ }^{17}$

\section{II.A.2. Sensitivity on Fusion Power}

To study the magnetic field strength and $\langle\beta\rangle$ within the constraints outlined above the magnetic field strength was varied continuously between $4 \mathrm{~T}$ and $6 \mathrm{~T}$ for the global power balance model for two constant $\langle\beta\rangle$ values. The first was $\langle\beta\rangle=3.6 \%$ 
as a lower bound and the other was $\langle\beta\rangle=4.9 \%$ as an upper bound, chosen to be close but still somewhat below the betalimit pointed out above. All other parameters were chosen as in HSR5/22 and kept constant except that the plasma density was increased along with the magnetic field in order to keep the value of $\langle\beta\rangle$ constant. The fusion power was chosen as the figure of merit for the plant performance. The results are illustrated in Fig. 2

It can be seen from Fig. 2 that at constant $\langle\beta\rangle$, the fusion power increases substantially with increasing magnetic field strength. The full step from NbTi technology with $B_{t}=4.5 \mathrm{~T}$ to $\mathrm{Nb}_{3} \mathrm{Sn}$ or $\mathrm{Nb}_{3} \mathrm{Al}$ technology with $B_{t}=5.6 \mathrm{~T}$ roughly doubles the fusion power. A vertical step from $\langle\beta\rangle=3.6 \%$ (solid line) to $\langle\beta\rangle=4.9 \%$ (dashed line) also nearly doubles the generated fusion power. If both the magnetic field strength and $\langle\beta\rangle$ can be increased simultaneously then a substantial gain in fusion power is achieved.

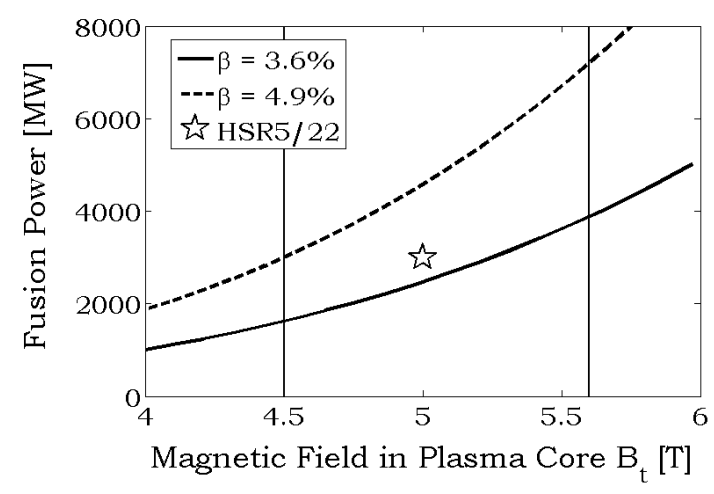

Fig. 2: Fusion power as function of the magnetic field strength for HSR5/22 parameters with constant $\langle\beta\rangle=3.6 \%$ (solid line) and $\langle\beta\rangle=4.9 \%$ (dashed line) where the horizontal line on the left is the maximum field strength of $\mathrm{NbTi}$ technology at $4.2 \mathrm{~K}$ and the limit on the right is the maximum field strength of $\mathrm{Nb}_{3} \mathrm{Sn}$ superconductors at $4.2 \mathrm{~K}$. The star-like symbol is the reference of the HSR5/22 point design. ${ }^{3}$

\section{II.B The Renormalisation Factor (0-D)}

The ISS04 data set showed a distinct clustering of sub-sets when plotted in figures of any available stellarator-heliotron scaling. ${ }^{7}$ This clustering motivated introduction of a sub-set dependent factor $\left(f_{\text {ren }}\right)$ which led to much more statistically significant scaling in $(a, R, n, P, B, t) . f_{\text {ren }}$ has been determined from reference scalings and is comparable to $H$-factors in tokamak scalings. It has been interpreted as reflecting configuration dependent confinement properties, but, although evidence for this interpretation has been found, it has not been conclusively proven. ${ }^{7}$ On the other hand, employing $f_{\text {ren }}$ to account for confinement enhancement effects appears to be a fairly obvious approach for the model discussed in this paper.

Therefore $f_{\text {ren }}$ was varied between $0-2$ and the impact on the machine size was investigated. More precisely, the size is the minimum major radius $R_{i g n}$ necessary to achieve ignition, which is defined as $Q=\infty$ and $P_{\text {heat }}=0$. The results are illustrated in Fig. 3 for a constant fusion power of $3 \mathrm{GW}$ and for both the 4 and 5-periodic HELIAS (solid and dashed line respectively). As the increase of $f_{\text {ren }}$ reflects improved confinement and in order to keep the fusion power constant, $\langle\beta\rangle$ is increased in line with $f_{\text {ren }}$.

Fig. 3 clearly shows that a confinement enhancement, especially around $f_{\text {ren }}=1$, allows for a reduction in the required device size. But as $\langle\beta\rangle$ is increased with $f_{\text {ren }}$ at one point a beta-limit is reached above which further confinement enhancement is pointless. This is shown in Fig. 3 with the circles at $\langle\beta\rangle=6 \%$. That means for the 5-periodic case a confinement enhancement with respect to the ISS04 above 1.7 is not meaningful with a corresponding minimum major radius of $R_{i g n}=17 \mathrm{~m}$. For the 4-periodic concept already an enhancement factor of about 1.5 reaches the beta-limit at a machine size of $R_{i g n}=13 \mathrm{~m}$. Another remarkable result of the study is that a minimum $f_{\text {ren }}=0.5$ seems to exist which precludes ignition for smaller values (for reasonable machine sizes $R_{0}<30 \mathrm{~m}$ ).

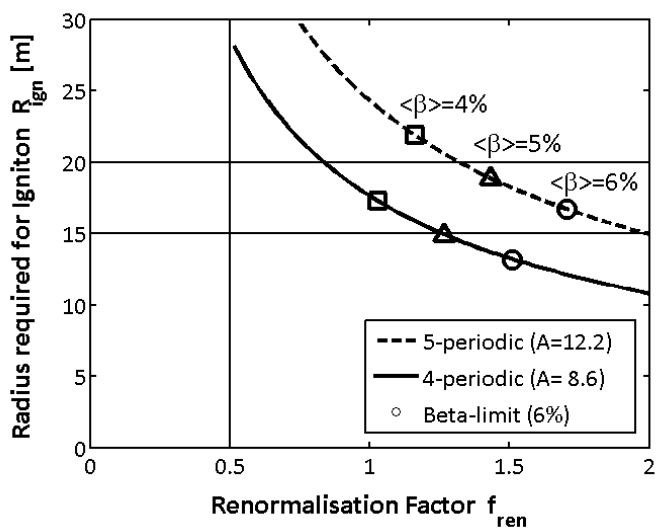

Fig. 3: The minimum major radius for plasma ignition $(Q=\infty)$ is shown in dependence of the renormalisation factor $f_{\text {ren }}$ for the 4 and 5-periodic HELIAS concepts (solid and dashed line respectively) at constant fusion power of $3 \mathrm{GW}$. As $\langle\beta\rangle$ is increased with $f_{\text {ren }}$, the circles show the point where $\langle\beta\rangle=6 \%$ is reached. Additionally, $\langle\beta\rangle=4 \%$ (squares) and $\langle\beta\rangle=5 \%$ (triangles) is shown.

From this result it is evident that the improvement and optimisation of the confinement is critical. There exists a minimum value of the confinement that must be achieved to reach ignition conditions at all, while, a very high degree of confinement allows for the reduction of device sizes. Such an improvement allows higher plasma temperatures and densities and is; therefore, interconnected to the plasma $\langle\beta\rangle$, which must be increased (up to the maximum possible value) along with the confinement improvement to truly allow for smaller devices. It is noted that, at the same time, further issues may result from particle confinement and density control. ${ }^{18}$ These topics will be treated elsewhere.

\section{II.C Direct Extrapolation of Wendeltein 7-X (0-D)}

Employing the introduced power balance model under the physics and engineering constraints listed in subsection II.A and additionally considering possible confinement enhancement with the renormalisation factor as stated above, Wendelstein 7-X can be directly extrapolated to power plant conditions. For this extrapolation two boundary scenarios are used. First a 
more conservative approach with a magnetic field strength of $B_{t}=4.5 \mathrm{~T}$ and line-averaged density $\bar{n}_{e}=1.8 \cdot 10^{20} \mathrm{~m}^{-3}$, and second a more advanced scenario with $B_{t}=5.5 \mathrm{~T}$ and $\bar{n}_{e}=2.8 \cdot 10^{20} \mathrm{~m}^{-3}$. Stellarators do not exhibit the strong Greenwald density limit observed in tokamaks. ${ }^{19}$ Nevertheless, a radiative density limit, known as SUDO-limit, ${ }^{20}$ has been observed in some heliotron/stellarator-type devices. However, the Large Helical Device (LHD) demonstrated the ability to operate far beyond this limit, especially if pellet injection is used. Therefore the SUDO-limit has been reinterpreted as a density limit for the plasma edge. ${ }^{21,22}$ For the 5-field period case the SUDO-limit yields a value of $1.6 \cdot 10^{20} \mathrm{~m}^{-3}$ which is very high for an edge-limit. The scenarios investigated in this work are well below these limits and were chosen to be somewhat below the corresponding ECRH cut-off density of the respective field strength assuming O1-mode heating.

For both scenarios W7-X is linearly scaled up at constant aspect ratio $A=10.3$ with the requirement to yield a fusion power of $P_{f u s}=3000 \mathrm{MW}$. Under these constraints a corresponding renormalisation factor is found which is needed to fulfill these conditions. The results for both scenarios are shown in Fig. 4 If $\mathrm{W} 7-\mathrm{X}$ is scaled up by factor 4 to $R_{0}=22 \mathrm{~m}$ major radius, the required renormalisation factor for the 'advanced' case is below 1 meaning that the $3 \mathrm{GW}$ fusion power can be reached without further confinement enhancement. In the 'conservative' case on the other hand, already a confinement enhancement factor of about 1.2 is required to achieve the necessary conditions for the $22 \mathrm{~m}$ machine. Looking next at upscaling of W7-X by a factor 3 , i.e. $16.5 \mathrm{~m}$ major radius, already the 'advanced' scenario needs a renormalisation factor of about 1.2 while the 'conservative' scenario would need a confinement enhancement factor of 1.8 which is here beyond the beta-limit of $\langle\beta\rangle=6 \%$ and therefore not realistically accessible. Again, $\langle\beta\rangle$ is increased parallel to $f_{\text {ren }}$ and in the case of an upscaled W7-X, the confinement enhancement beyond $f_{\text {ren }}=1.6$ becomes restricted by the beta-limit.

\section{PREDICTIVE NEOCLASSICAL TRANSPORT MODEL}

The 3D magnetic field of stellarators introduces a large class of localised helical trapped particle orbits which leads to socalled 'neoclassical' transport. This is a drawback of the stellarator as the resulting particle and energy fluxes are much higher than in tokamaks. Nonetheless, these effects and the corresponding transport are well understood ${ }^{23,24}$ and even allow to predict the stellarator-specific ambipolar electric field. These physics based predictions are especially relevant for larger machines with higher temperatures since the neoclassical transport scales strongly with temperature.

The neoclassical transport model, which will be introduced below, includes profile effects and especially allows to selfconsistently calculate the plasma transport and the corresponding confinement time. Two scenarios, one employing conservative and one advanced physics and technology assumptions are simulated by upscaling the W7-X high-mirror configuration. By comparing the calculated confinement time to the

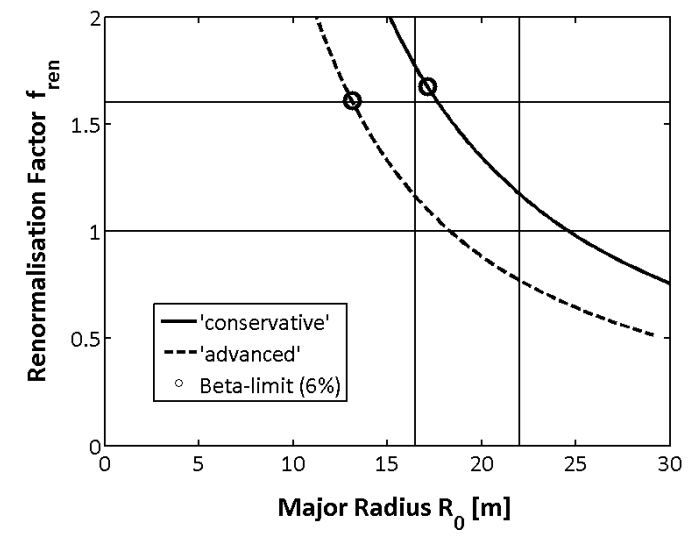

Fig. 4: The required renormalisation factor $f_{\text {ren }}$ with respect to the ISS04 confinement time scaling is shown for linear extrapolation of Wendelstein 7-X with major radius at fixed aspect $A=10.3$ ratio and fusion power $P_{\text {fus }}=3000 \mathrm{MW}$. Two scenarios are illustrated, a 'conservative' one with $B_{t}=4.5 \mathrm{~T}$ and $\bar{n}_{e}=1.8 \cdot 10^{20} \mathrm{~m}^{-3}$ (solid line) and an 'advanced' one with $B_{t}=5.5 \mathrm{~T}$ and $\bar{n}_{e}=2.8 \cdot 10^{20} \mathrm{~m}^{-3}$ (dashed line). As again $\langle\beta\rangle$ is increased with $f_{\text {ren }}$ the circles show the point where $\langle\beta\rangle=6 \%$ is reached.

ISS04 scaling a predictive $f_{\text {ren }}^{1 \mathrm{D}}$ can be obtained. The last section showed the critical impact of $f_{\text {ren }}$, so the 1-D simulations permit an assessment of the current optimisation of the HELIAS (upscaled W7-X high mirror configuration) with respect to the ISS04. It should be noted that further optimisation of stellarator magnetic configurations is the subject of on-going research. ${ }^{25,26}$

In the predictive neoclassical transport model $27,28,29,30$ it is assumed that the plasma may be described by using a local approximation to solve the drift kinetic equation. Additional fluxsurface averaging allows reduction of the geometrical description of the plasma to one coordinate, namely the flux surface label $\psi$ that can be related to the average minor plasma radius $r=\sqrt{\psi / \psi_{\max }}$. With this radial coordinate the power balance of the plasma is expressed for the electrons $s=e$ and ions $s=i$ by the equations

$$
\frac{3}{2} \frac{\partial n_{s} T_{s}}{\partial t}+\frac{1}{V^{\prime}} \frac{\partial}{\partial r}\left(V^{\prime} Q_{s}\right)=P_{s}+Z_{s} \Gamma_{s} E_{r}
$$

with the power source / loss term $P_{s}$ consisting of heating power, bremsstrahlung and the collisional coupling between electrons and ions. The neoclassical particle, $\Gamma_{s}$, and energy, $Q_{s}$, fluxes are expressed ${ }^{24}$ as:

$$
\begin{aligned}
& \Gamma_{s}=-n_{s} L_{11}^{s}\left[\frac{n_{s}^{\prime}}{n_{s}}-\frac{Z_{s} e E_{r}}{T_{s}}+\left(\frac{L_{12}^{s}}{L_{11}^{s}}-\frac{3}{2}\right) \frac{T_{s}^{\prime}}{T_{s}}\right] \\
& Q_{s}=-n_{s} T_{s} L_{21}^{s}\left[\frac{n_{s}^{\prime}}{n_{s}}-\frac{Z_{s} e E_{r}}{T_{s}}+\left(\frac{L_{22}^{s}}{L_{21}^{s}}-\frac{3}{2}\right) \frac{T_{s}^{\prime}}{T_{s}}\right]
\end{aligned}
$$

where the thermal transport coefficients $L_{i j}$ are obtained by the appropriate energy convolutions with the local Maxwellian distribution function

$$
L_{i j}=\frac{2}{\sqrt{\pi}} \int_{0}^{\infty} \sqrt{K} e^{-K} D_{\perp}(K) h_{i} h_{j} \mathrm{~d} K
$$


with $K=m v^{2} / 2 T, h_{1}=1, h_{2}=K$ of interpolated results for $D_{\perp}$ from a full mono-energetic diffusion coefficient database $\mathrm{e}^{31,32}$

$$
D_{\perp}=D_{\perp}\left(\rho, \frac{R_{0} v_{s}}{v_{s} \iota}, \frac{E_{r}}{v_{s} B_{0}}\right) .
$$

Here $v_{s}, v_{s}, Z_{s}$ are the collision frequency, velocity and charge number of electrons or ions, $B_{0}$ the value of the magnetic field and $E_{r}$ the radial electric field.

Usually, the ambipolarity constraint $Z_{i} \Gamma_{i}=\Gamma_{e}$ is solved to obtain the radial electric field $E_{r}$. However, this approach is often not numerically suitable because of the discontinuity and the bifurcations of the solution. This problem can be circumvented with a diffusion equation for the radial electric field from the poloidal force balance: ${ }^{33}$

$$
\frac{\partial E_{r}}{\partial t}-\frac{1}{V^{\prime}} \frac{\partial}{\partial r}\left(D_{E} V^{\prime} r \frac{\partial}{\partial r}\left(\frac{E_{r}}{r}\right)\right)=\frac{|e|}{\epsilon}\left(\Gamma_{e}-Z_{i} \Gamma_{i}\right)
$$

with $D_{E}$ the 'diffusion coefficient' of the electric field and the dielectric constant $\epsilon$. This is necessary as localised central heating (e.g. with ECRH) can lead to high electron and low ion temperatures amplifying electron transport. The solution of the ambipolarity constraint then becomes the so-called 'electronroot' with a strong, positive electric field in the centre while the edge with similar electron and ion temperatures is governed by the 'ion-root' solution with a negative electric field. Eq. (12) for the electric field allows then for a smooth crossing of $E_{r}$ from the 'electron' to 'ion-root', especially where several solutions of the ambipolarity constraint coexist.

Due to the strong temperature dependence of the neoclassical transport stellarators are, especially in the plasma centre, dominated by neoclassical transport effects where the temperature is highest. Turbulence is assumed to play only a subdominant role in the centre because of high neoclassical diffusion coefficients. The plasma centre has a higher temperature than the plasma edge and; therefore, the neoclassical transport becomes small at the edge so that the anomalous transport phenomena starts to dominate. This has been observed in many experiments, e.g. ${ }^{23}$ This results in the requirement that to the neoclassical description the additional anomalous transport must be accounted for. As models based on first principles for anomalous transport are not yet available, a basic experimentally derived model is employed with:

$$
Q_{s}^{a n o}=-\chi_{s}^{a n o} n_{s} T_{s}^{\prime}
$$

where the anomalous heat conductivity $\chi \sim P^{3 / 4} n^{-1}$ is taken from experimental results obtained in W7-AS. ${ }^{34,35}$ The discussion of anomalous transport in 3D configurations is the subject of on-going research. ${ }^{36}$ To what extent Eq. 13 represents an adequate description is not yet determined.

The neoclassical treatment, on the other hand, as described here has been extensively validated with experiments ${ }^{23}$ and the corresponding codes have been benchmarked in detail. ${ }^{24}$

\section{III.A Direct Extrapolation of Wendeltein 7-X (1-D)}

In the following the two scenarios from subsection II.C are taken up again and refined in more detail: one assumes conservative and the other advanced physics and technology assumptions, explicitly defined in Tab. I] Both scenarios employ the W7-X high-mirror magnetic configuration linearly upscaled from a major radius of 5.5 to that of $22 \mathrm{~m}$. The impact of these assumptions on fusion power, fusion gain, and the calculated confinement time are investigated. As fueling and exhaust scenarios are beyond the scope of this work, both scenarios were simulated using a flat density profile. Although the 1-D model is capable of self-consistently treat the density profile with respect to fuelling, in this work the profile was held constant and detailed fuelling scenarios will be a topic of future investigations.

The conservative scenario assumes $\mathrm{NbTi}$ superconductor technology (at $4.2 \mathrm{~K}$ ) with $4.5 \mathrm{~T}$ magnetic field on axis. The line averaged electron density is chosen to be moderate with $\bar{n}_{e} \approx 1.8 \cdot 10^{20} \mathrm{~m}^{-3}$. On the one hand this leads to a smaller $\langle\beta\rangle$ and as shown in the previous chapter, this implies a lower fusion power. In the advanced scenario, the technologically advanced $\mathrm{Nb}_{3} \mathrm{Sn}$ superconductor technology is assumed (at $4.2 \mathrm{~K}$ ) with 5.5 $\mathrm{T}$ field strength on axis. The density is also chosen higher with $\bar{n}_{e} \approx 2.8 \cdot 10^{20} \mathrm{~m}^{-3}$.

Both densities were selected to be somewhat below the ECRH cut-off density to allow for controlled heating. The W7$\mathrm{X}$ high-mirror configuration has a mirror term of the magnetic field strength of around $10 \%$ in the plasma centre. This provides a highly localised resonance for O1-mode ECRH heating at $B_{\max }$ (including mirror term) near the magnetic axis. In the conservative case with $B_{0}=4.5 \mathrm{~T}$ on axis, the W7-X $140 \mathrm{GHz}$ gyrotrons are then applicable which have the resonance at $B=5 \mathrm{~T}$ and for the advanced scenario with $B_{0}=5.5 \mathrm{~T}$ the $170 \mathrm{GHz}$ ITER gyrotrons would be the choice with the resonance at $B=6 \mathrm{~T}$. Therefore the ECRH absorption profile is modelled by a Gaussian shape assuming that the full power can under resonant conditions be deposited in the plasma centre.

The two scenarios introduced above were simulated within the predictive neoclassical transport model by an upscaling of the magnetic configuration for major radii between 5.5 and $22 \mathrm{~m}$. Fusion power and $\alpha$-heating were calculated selfconsistently. The fusion power and the fusion gain $Q=$ $P_{\text {fus }} / P_{\text {heat }}$ serve as figures of merit where the external ECRH heating was continuously reduced while going to larger device size until ignition is reached $(Q=\infty)$. The results are illustrated in Fig. 5. It can be seen that the fusion power increases strongly with machine size (left side of Fig. 5p. This increase is due to the growth of the plasma volume with device size. As the field and density are fixed within one scenario, the enhancement of the plasma volume increases the total number of particles and; therefore, the number of fusion reactions, and thus fusion power.

It is also evident that the step from the conservative to the advanced assumptions, with higher density and magnetic field increases the fusion power even more. The enhanced confinement due to the stronger magnetic field results in a higher density of particles leading to a higher plasma pressure and strong increase in fusion power. These findings confirm the results of the global power balance approach.

As the fusion power increases strongly with increasing machine size the need for external heating is correspondingly reduced which was consequently decreased in the simulations. 


\begin{tabular}{llllll} 
Scenario & $\begin{array}{l}\text { Config- } \\
\text { uration }\end{array}$ & $\begin{array}{l}\mathrm{SC} \text { Tech- } \\
\text { nology }\end{array}$ & $B_{0}[\mathrm{~T}]$ & $\bar{n}_{e}\left[10^{20} \mathrm{~m}^{-3}\right]$ & Gyrotrons \\
\hline conservative & $\begin{array}{l}\text { W7-X } \\
\text { high } \\
\text { mirror }\end{array}$ & $\mathrm{NbTi}$ & 4.5 & 1.8 & W7-X: $140 \mathrm{GHz}$ \\
advanced & $\begin{array}{l}\text { W7-X } \\
\text { high } \\
\text { mirror }\end{array}$ & $\mathrm{Nb}_{3} \mathrm{Sn}$ & 5.5 & 2.8 & ITER: $170 \mathrm{GHz}$ \\
& & & & \\
\hline
\end{tabular}

TABLE III: Summary of the parameters of the conservative and advanced scenario.

Accordingly the fusion gain, which is the ratio of fusion power over external heating, strongly increases with increasing major radius as can be observed on the right side of Fig. 5 until reaching ignition (the lines end as $Q \rightarrow \infty$ ).
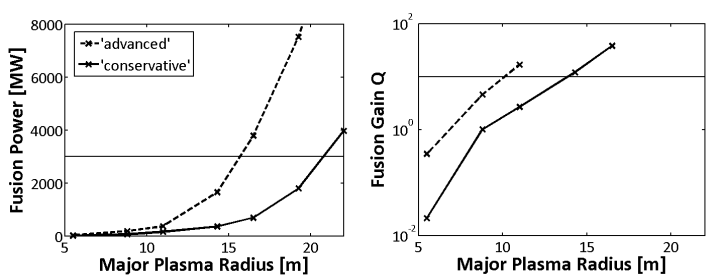

Fig. 5: Self-consistent fusion power (left) and fusion gain (right) for the conservative (solid line) and advanced scenario (dashed line).

At a first glance the advanced scenarios seem capable of drastically reducing the device size. These smaller sizes should be seen as the lowest limit practicably achievable under the advanced physics and engineering conditions. But several points are not considered in the simulations which violate the idealised assumptions. These are discussed in the following.

The anomalous (or turbulent) transport has been treated in the predictive simulations only by a simple scaling model based on experimental results. Although theory suggests that some turbulent micro instabilities may be stabilised in stellarators, e.g. trapped electron modes, others like the ion temperature gradient driven modes may contribute more to the overall plasma transport then accounted for by the simple model ${ }^{37}$ used in this paper. Transport and thus the confinement time may; therefore, be less favourable than the simulations suggest.

\section{III.B The Renormalisation Factor (1-D)}

As stated in section II.B, one important figure of merit to characterise plasma transport is the energy confinement time. The neoclassical transport simulations for the 'conservative' and 'advanced' scenario (cf. previous section) also provided predictive confinement times. With these predictions it is possible to characterise the confinement enhancement found in the simulations by comparison with the empirical ISS04 scaling law. This is done by defining the factor

$$
\Theta=\tau_{E}^{1 \mathrm{D}} / \tau_{E}^{\mathrm{ISS} 04}
$$

which is the ratio of the simulated confinement time over the corresponding ISS04 value, i.e. the confinement enhancement factor obtained from the predictive simulations. In that sense it could be interpreted as a $1 \mathrm{D}$ renormalisation factor $f_{\text {ren }}^{1 \mathrm{D}}$. The label $\Theta$ is chosen to better distinguish the results from the ones obtained for the 0 -D power balance model.

The results are illustrated in Fig. 6 where $\Theta$ is plotted against the plasma volume for the 'conservative' as well as the 'advanced' scenario. It can be seen that $\Theta$ is not a constant factor but decreases when extrapolated to reactor conditions. For both the conservative and the advanced scenario, $\Theta$ changes from around 2 (W7-X size) down to $1.1-1.3$ (HELIAS reactor size). If $\Theta$ is interpreted to be a confinement enhancement factor similar to the renormalisation factor $f_{r e n}$, then this is a large change when compared to the results from the global power balance model (Fig. 3).

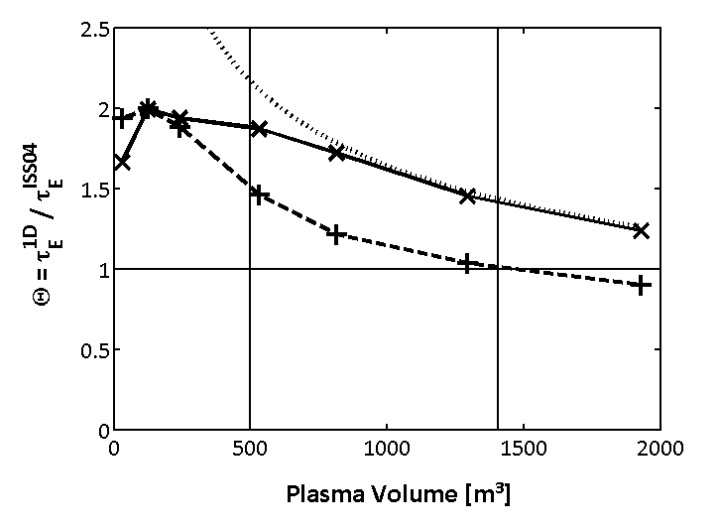

Fig. 6: Confinement time of the predictive neoclassical simulations normalised to the ISS04 scaling for the 'conservative' scenario (solid line) compared to a normalised $\Theta \sim V^{-2 / 5}$ scaling (dotted line), as well as the 'advanced' scenario (dashed line). The vertical solid lines represent $Q \geq 10$ (left) and HSR5/22 in terms of plasma volume (right).

Before the results are explained, some general remarks regarding confinement scaling laws are necessary in order to avoid unphysical interpretation of the given results. Empirical scalings may accurately describe the available data range but extrapolation outside this range is beyond the validity of the scaling. The used expressions and their combination is not unique and rephrasing with respect to other parameters is possible.

Some insight in the behaviour of the confinement enhancement factor, $\Theta$, may be gained by revisiting the definition of the energy confinement time $\tau_{E}=W / P$. The energy can be expressed by $W=V \beta B^{2}$, Eq. (3), and the lost power with $P=P_{\alpha}-P_{\text {brems }}+P_{E x t}$ according to section III Additionally, 
it is assumed that bremsstrahlung $P_{\text {brems }}$ only occurs under reactor conditions with usually $P_{b r e m s} / P_{\alpha} \approx 15 \%$ and therefore $P_{\alpha} \gg P_{\text {brems }}$. Neglecting bremsstrahlung, the energy confinement time is

$$
\tau_{E}=\frac{W}{P} \sim \frac{V \beta B^{2}}{P_{\alpha}+P_{E x t}}
$$

where $P_{E x t}$ is the externally applied heating power. The alpha heating power is $1 / 5$ of the fusion power that may be approximated by $P_{\alpha} \sim V \beta^{2} B^{4}$ as given by Eq. (5). Inserting in Eq. (15) leads to

$$
\tau_{E} \sim \frac{V \beta B^{2}}{V \beta^{2} B^{4}+P_{E x t}} .
$$

From this representation it becomes clear that the confinement time consists of two different parts. The first part is the influence of the alpha heating power on the confinement time, while the second part comes from the externally applied heating. The behaviour of $\tau_{E}$ will be different depending on which of these two is dominant and it is therefore useful to consider two distinct asymptotic cases:

i) No fusion power: $P_{\alpha}=0$

$$
\Rightarrow \tau_{E}^{E x t} \sim \frac{V \beta B^{2}}{P_{E x t}}
$$

This case corresponds to the situation without fusion power and no significant bremsstrahlung. The externally given heating power $P_{E x t}$ is thus a free parameter. This together with the clear volume dependence is characteristic of the regime in which the empirical confinement time scaling ISSO4 was derived.

ii) High fusion power: $P_{\alpha} \gg P_{E x t}$

$$
\Rightarrow \tau_{E}^{\alpha} \sim \frac{1}{\beta B^{2}}
$$

In this case the external heating is neglected and only alpha particles heat plasma. The heating power is, therefore, no longer a free parameter. Instead, it is interconnected to the plasma volume, beta, and field: $P_{\alpha} \sim V \beta^{2} B^{4}$. As such, $\tau_{E}$ scales in this representation differently than in case $i$ ).

With this analysis, the confinement time of the fusion case $\tau_{E}^{\alpha}$ can be compared with the ISS04 scaling law by inserting the alpha power $P_{\alpha} \sim V \beta^{2} B^{4}$ in the ISS04 $\tau_{E}^{\mathrm{ISS} 04} \sim V n^{3 / 5} B^{4 / 5} P_{\alpha}^{-3 / 5}$ leading to

$$
\tau_{E}^{\mathrm{ISS} 04, \alpha} \sim \frac{V^{2 / 5} n^{3 / 5}}{\beta^{6 / 5} B^{8 / 5}} .
$$

Finally, the enhancement factor $\Theta$ for the high fusion power case can be approximated with $\Theta^{\alpha}=\tau_{E}^{\alpha} / \tau_{E}^{\text {ISS04, } \alpha}$ resulting in

$$
\Theta^{\alpha} \sim \frac{\beta^{1 / 5}}{V^{2 / 5} n^{3 / 5} B^{2 / 5}} .
$$

As the specific plasma transport and transport regimes play only a role in the achievable $\beta$ and because $n$ and $B$ were held constant in the predictive transport simulations, it can be concluded that the degradation of the confinement enhancement factor $\Theta$ with respect to the ISSO4 is directly related to the increase of the volume in the high fusion power scenario

$$
\Theta^{\alpha} \sim V^{-2 / 5} \text {. }
$$

This relation is plotted in Fig. 6and agrees with the simulations at high fusion power giving an indication that the decrease of $\Theta$ may be related to the fact that the heating power is determined self-consistently by machine and plasma performance parameters.

\section{III.C Impact of Transport on the Renormalisation Factor}

In the last subsection a basic scaling approach was used to conceptually obtain a relation for the decrease of the confinement enhancement factor $\Theta$. In the following a basic analysis of the underlying transport mechanism shall be given which plays an important role for the achievable plasma performance.

\section{III.C.1. Scaling Considerations}

Considering first the empirical confinement time scaling ISS04, the majority of data points used for the regression of the scaling have been obtained at moderate to high collisionality, $v^{*}=O\left(10^{-1}\right)$, corresponding to the neoclassical plateau regime. The analytic limit of the neoclassical plateau regime leads to a confinement time scaling which closely resembles the exponents of the ISS04:

$$
\tau_{E}^{n e o, P l} \propto \frac{n T}{P} \propto n^{3 / 5} P^{-3 / 5} B^{4 / 5} \epsilon^{2 / 5}
$$

Although the neoclassical scaling agrees with the ISS04, in W7AS, the energy and particle fluxes could not be explained by the neoclassical plateau regime as the fluxes were systematically underestimated. Only a few high-performance discharges of the database at $T \geq 1 \mathrm{keV}$ matched the predicted neoclassical fluxes. In this context it should be noted, that a gyro-Bohm type turbulence approach for tokamaks, the so-called LacknerGottardi scaling, ${ }^{38}$ leads to the same exponential relations as exhibited by the ISS04 and neoclassical plateau regime scaling.

These basic scaling considerations already raise doubts about the usage of the ISSO4 scaling for extrapolation to stellarator power plant conditions. This is especially true in the regime of a burning plasma with self-sustained alpha heating as shown in the last subsection where the dimensionless parameters $\rho^{*}, v^{*}$, and $\beta$ are far outside the experimental results. The similartiy principle requires that the dimensionless quantities describing a geometrical similar system must be the same to allow extrapolation which is violated here.

\section{III.C.2. Neoclassical Transport Considerations}

The neoclassical transport coefficients have different scaling properties dependent on the collisionallity regime. In the upscaling of the 'conservative' as well as in the 'advanced' simulation scenario the core temperature increases with increasing 
machine size. This in turn means that the collisionallity is decreasing. Therefore, at increasing machine size both the electron and ion distribution funcion will shift to neoclassical longmean-free-path regimes ( $1 / v$ for electrons and $\sqrt{v}$ for ions). Due to the localised particles, the neoclassical diffusion coefficients in these regimes are an order of magnitude higher for the stellarator than for a corresponding tokamak. The neoclassical theory is also relevant for small stellarators (e.g. W7-AS) where easily $D^{\text {neo }}>1 \mathrm{~m}^{2} / \mathrm{s}$. It should also be noted, that the electrons are well confined in an optimised stellarator, meaning the ion losses would be much larger than those for the electrons. This is prevented by the ambiporaity constraint which causes an inward pointing radial electric field bringing the ion transport down to the electron level while on the other hand somewhat increasing the electron transport.

This complex combination of different transport regimes with distinct scaling relations and additional coupling by the ambipolar radial electric field precludes the derivation of a combined analytic confinement time scaling which can only be given for individual regimes. This means, that although empirical scalings may be derived by regression, the confinement time scales differently dependent on the plasma properties.

\section{III.C.3. Anomalous Transport Considerations}

To complicate the situation even more, also the anomalous transport needs to be taken into account. As of today's experiments the anomalous transport has been observed to dominate at the plasma edge. Following this observation the empirical relationship $\chi_{\text {ano }}=c P^{3 / 4} n^{-1}$ has been employed for the anomalous transport in the 1-D simulations where the prefactor $c$ is a free variable. In the upscaling simulations for both the 'conservative' and 'advanced' scenario the factor was fixed to $c=1$. But the strong power dependence of this simple model leads to very high anomalous transport for larger machine sizes which seems from today's experiments unlikely. For this reason one simulation has been repeated with reduced $c$ to investigate the general sensitivity. This is illustrated in Fig. 7, where the ratio of the ion neoclassical to anomalous energy flux is given over the normalised minor radius for the 'conservative' scenario at machine size $V=1300 \mathrm{~m}^{3}$ for $c=1$ and $c=0.2$ (selected to achieve $\chi_{\text {ano }}=1 \mathrm{~m}^{2} / \mathrm{s}$ at the edge observed in many experiments compared to $\chi_{\text {ano }}=2.5 \mathrm{~m}^{2} / \mathrm{s}$ for the $c=1$ case).

It can be seen from the figure that in the case with the reduced prefactor, $c=0.2$, the anomalous transport is consequently reduced and the neoclassical transport dominates over the greater part of the minor radius except at the very edge with $\chi_{\text {ano }}=1 \mathrm{~m}^{2} / \mathrm{s}$. In the case with the 'normal' prefactor, $c=1$, the neoclassical transport is still larger by a factor 4 in the plasma centre but an increased portion of the edge beyond $\rho \geq 0.7$ is dominated by the anomalous energy flux with $\chi_{\text {ano }}=2.5 \mathrm{~m}^{2} / \mathrm{s}$ at the very edge.

Comparing both cases, the prefactor has a strong impact on the plasma transport and performance as the plasma $\beta$, volume averaged temperature $\left\langle T_{i}\right\rangle_{V}$, and the resulting alpha power nearly doubles in the case with the reduced anomalous transport. But the confinement ratio factor $\Theta$, discussed in the previous subsection, changes only modestly from $\Theta=1.45$ in the

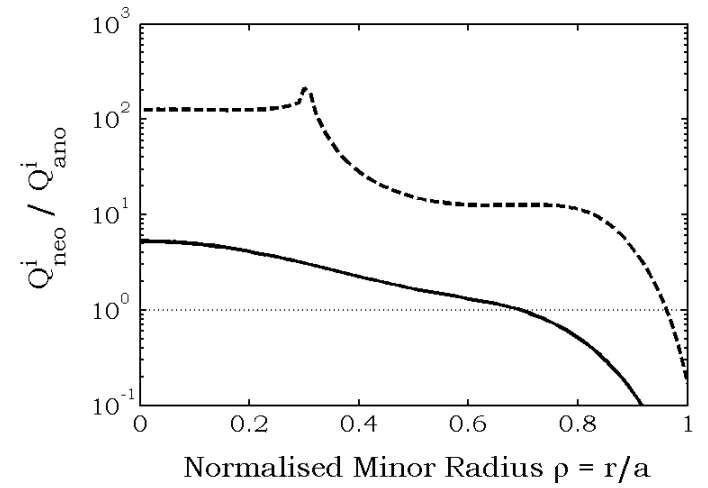

Fig. 7: Comparison of the ratio of ion neoclassical to anomalous heat flux, $Q_{\text {neo }}^{i} / Q_{\text {ano }}^{i}$, over normalised minor radius, $\rho=r / a$, for the 'conservative' scenario at machine size $V=1300 \mathrm{~m}^{3}$ for two different prefactors in the anomalous transport model: $c=1$ (solid line) and $c=0.2$ (dashed line).

case with $c=1$ to $\Theta=1.62$ with the reduced anomalous transport $c=0.2$. Also, due to the impact of the anomalous transport, the lines in Fig. 7 exhibit different shape. The reason is, that the increased anomalous transport reduces the temperature of the plasma leading to reduced fusion power and alpha heating. As the neoclassical transport is very sensitive to temperature in the lmfp-regime $\left(D \sim T^{7 / 2}\right.$ for $\left.1 / v\right)$, neoclassical transport is reduced at increasing anomalous transport. The complex interplay with the electric field then leads to the strongly different ratios of neoclassical to anomalous ion energy flux.

It can be concluded that, apart from neoclassical transport, the anomalous transport plays an important role in reactor-sized HELIAS machines. A detailed understanding and extrapolation of anomalous, i.e. turbulent transport seems necessary to make precise predictions about the plasma performance of a burning plasma HELIAS. Although a simple empirical anomalous transport model was employed and gave first indications, the descriptive significance is limited. Turbulence simulations for 3-D stellarator geometries have been started recently. ${ }^{36}$ The aim of this investigation lies especially on the ion-temperaturegradient mode which is anticipated to be a dominant microinstability contributing to transport. From these studies, more relevant anomalous transport models may be derived compatible with the 1-D code increasing the general predictive capability.

\section{III.D Comparison of 0-D and 1-D Model}

For the conservative scenario the direct extrapolation of W7$\mathrm{X}$ within the 0-D model with $P_{f u s}=3000 \mathrm{MW}$ and the ISS04 confinement time scaling at $f_{\text {ren }}=1$ would lead to a machine with a major radius around $25 \mathrm{~m}$. The same conservative scenario achieves within the self-consistent 1-D transport model a fusion power of $3 \mathrm{GW}$ at a major radius of $21 \mathrm{~m}$ and reaches a confinement time a factor 1.3 above the ISS04 scaling. If this confinement enhancement factor of 1.3 is applied to the 0-D model a major radius of $21 \mathrm{~m}$ is found which is consistent with the transport model. 
In the advanced scenario the 0-D extrapolation for $f_{\text {ren }}=1$ yields a major radius of around $19 \mathrm{~m}$. In the transport simulations with the high density and field the conditions are already achieved at a upscaling of W7-X times 3, i.e. $16.5 \mathrm{~m}$ major radius, where a confinement time is achieved with a factor 1.2 beyond the ISS04 scaling. If this factor is again allowed for the 0 -D model then the $16.5 \mathrm{~m}$ major radius are likewise found. All values are summarised in Tab. IV

\begin{tabular}{llll} 
Model & $0-\mathrm{D}$ & $0-\mathrm{D}$ & $1-\mathrm{D}$ \\
\hline conservative & & & \\
Major Radius [m] & 25 & 21 & $21^{*}$ \\
Conf. Enhancement Factor & $1.0^{*}$ & $1.3^{*}$ & 1.3 \\
\hline advanced & & & \\
Major Radius [m] & 19 & 16.5 & $16.5^{*}$ \\
Conf. Enhancement Factor & $1.0^{*}$ & $1.2^{*}$ & 1.2 \\
\hline
\end{tabular}

TABLE IV: Summary of the major radius from direct extrapolation of Wendelstein 7-X with associated confinement enhancement factors for the comparison between the power balance model and the predictive transport model constraint by achieving $3000 \mathrm{MW}$ fusion power. The values marked with a star are the respective input parameters in the model.

This result is important, as it means, that empirical scalings like ISS04 cannot be straightforwardly taken to extrapolate today's machines to devices of the size of fusion power plants. Rather, extrapolations must be iterated with predictive transport simulations due to the high impact of confinement on plant performance. In this respect it is important to consider the details of the magnetic configuration which plays a major role for the energy confinement. Although this work concentrated on the helical-axis advanced stellarator line, similar effects can be expected from other stellarators as the driving underlying physics are the same.

Therefore, the renormalisation factor cannot be taken as a simple constant in combination with an empirical confinement time scaling, but in total must either be iterated with transport simulations or a new consistent scaling be derived.

Nonetheless, the consistency of the 0-D and the 1-D model is a promising result. It means, that indeed predictive transport simulations can be carried out to narrow down an achievable confinement enhancement factor which then in turn can be used for systematic studies in a more simplified model as e.g. the power balance model of systems codes - as long as the transport simulations are iterated with the corresponding parameters.

\section{CONCLUSIONS}

From both models it is concluded that the confinement enhancement with respect to the ISS04 scaling has on the one hand a lower limit of about 0.5 under which ignition is not possible anymore. On the other hand, it has an upper limit in the range $1.5-1.8$ which is determined by the beta-limit above which the confinement enhancement no longer contributes to higher machine performance. Moreover; from the transport simulations can be learned that a confinement enhancement factor cannot be arbitrarily assumed since the confinement time is self-consistently determined from machine parameters and the complex interplay of transport effects. For the extrapolation of $\mathrm{W} 7-\mathrm{X}$ to reactor conditions using the predictive transport model the confinement enhancement is found to be in the range of 1.2 - 1.3. This means, empirical scalings and constant renormalisation factors cannot be used to directly extrapolate to fusion power plant devices, but, rather extrapolations must be iterated with predictive transport simulations. All these conclusions are in contradiction with the common assumptions of many power plant studies where confinement enhancements factors are arbitrarily assumed up to a factor 2 in order to design compact machines. Results of these studies should be reassessed under the limits of confinement enhancement. It should be noted that similar simulations have also been carried out for a $\mathrm{He}$ liotron configuration which exhibited the same behaviour, but such work was not pursued in detail as the focus of this work is put on HELIAS devices.

In this work the W7-X high-mirror configuration has been upscaled. But optimisation procedures have progressed lately so that new quasi-isodynamic (minimum- $B$, maximum- $J$ ) configurations with poloidally closed contours of $B$ may be an option for the future which exhibit very low neoclassical transport and good confinement of fast particles. Additionally, new insight into occurrence of turbulent micro instabilities allows further optimisation of such advanced configurations for reduced anomalous transport. Such configurations are candidates for HELIAS power plants and achieve even higher confinement enhancement factors (with respect to the ISS04 scaling).

Finally it can be concluded from the consistency of the global power balance approach and the predictive transport model that systems codes (which employ power balance models) can indeed be used to design and study HELIAS power plants as long as the corresponding confinement times are provided iteratively from the detailed predictive transport simulations. This makes the iteration process more complex since $f_{\text {ren }}$ introduces new dependencies on all important parameters which must be considered for consistency. Nonetheless, with this approach new systematic studies of HELIAS burning plasma devices are to follow in the future.

\section{ACKNOWLEDGMENTS}

This work has been carried out within the framework of the EUROfusion Consortium and has received funding from the Euratom research and training programme 2014-2018 under grant agreement No 633053. The views and opinions expressed herein do not necessarily reflect those of the European Commission.

\section{REFERENCES}

${ }^{1}$ T. Goto, J. Miyazawa, H. Tamura et al. "Design Window Analysis for the Helical DEMO Reactor FFHR-d1." Plasma and Fusion Research: Regular Articles, vol. 7, p. 2405084 (2012).

${ }^{2}$ C. D. Beidler, E. Harmeyer, F. Herrnegger et al. "The Helias reactor HSR4/18." Nuclear Fusion, vol. 41, p. 1759 (2001).

${ }^{3}$ J. Kisslinger, ed. Fusion energy Proc. 17th Int. Conf., vol. 4 of 1239-42. IAEA, Vienna, Yokohama (1998). 
${ }^{4}$ F. Warmer, C. D. Beidler, A. Dinklage et al. "HELIAS Module Development for Systems Codes.” Fusion Engineering and Design, vol. 91, p. 60 (2014). ${ }^{5}$ F. Warmer, C. D. Beidler, A. Dinklage et al. "Implementation and Verification of a HELIAS module for the Systems Code PROCESS." Fusion Engineering and Design, vol. [in press] (2014).

${ }^{6}$ H. Zohm. "On the minimum size of DEMO." Fusion Science and Technology, vol. 58, p. 613 (2010).

${ }^{7}$ H. Yamada, J. H. Harris, A. Dinklage et al. "Characterization of energy confinement in net-current free plasmas using the extended International Stellarator Database." Nuclear Fusion, vol. 45, p. 1684 (2005).

${ }^{8}$ A. Dinklage, H. Maßßberg, R. Preuss et al. "Physical model assessment of the energy confinement time scaling in stellarators." Nuclear Fusion, vol. 47, p. 1265 (2007).

${ }^{9}$ H. Wobig, T. Andreeva, C. D. Beidler et al. "Concept of Helias ignition experiment." Nuclear Fusion, vol. 43, p. 889 (2003).

${ }^{10}$ F. Schauer. "Coil winding pack FE-analysis for a HELIAS reactor." Fusion Engineering and Design, vol. 86, p. 636 (2011).

${ }^{11}$ F. Schauer, K. Egorov and V. Bykov. "HELIAS 5-B magnet system structure and maintenance concept." Fusion Engineering and Design, vol. 88, p. 1619 (2013).

12 W. Lotz, P. Merkel, J. Nührenberg et al. "Collisionless alpha -particle confinement in stellarators." Plasma Physics and Controlled Fusion, vol. 34, p. 1037 (1992).

${ }^{13}$ H. Yamada, A. Komori, N. Ohyabu et al. "Configuration flexibility and extended regimes in Large Helical Device." Plasma Physics and Controlled Fusion, vol. 43, no. 12A, p. A55 (2001).

${ }^{14}$ A. Weller, J. Geiger, A. Werner et al. "Experiments close to the beta-limit in W7-AS." Plasma Physics and Controlled Fusion, vol. 45, no. 12A, p. A285 (2003).

15 W. A. Cooper, L. Brocher, J. P. Graves et al. "Drift Stabilisation of Ballooning Modes in an Inward-Shifted LHD Configuration." Contributions to Plasma Physics, vol. 50, p. 713 (2010).

${ }^{16} \mathrm{~K}$. Ichiguchi and B. A. Carreras. "Multi-scale MHD analysis incorporating pressure transport equation for beta-increasing LHD plasma." Nuclear Fusion, vol. 51, no. 5, p. 053021 (2011).

${ }^{17}$ M. Drevlak, D. Monticello and A. Reiman. "PIES free boundary stellarator equilibria with improved initial conditions." Nuclear Fusion, vol. 45, p. 731 (2005).

${ }^{18}$ H. Maaßberg, C. D. Beidler and E. E. Simmet. "Density control problems in large stellarators with neoclassical transport." Plasma Physics and Controlled Fusion, vol. 41, p. 1135 (1999).

${ }^{19}$ M. Greenwald. "Density limits in toroidal plasmas." Plasma Physics and Controlled Fusion, vol. 44, no. 8, p. R27 (2002).

${ }^{20}$ S. Sudo, Y. Takeiri, H. Zushi et al. "Scalings of energy confinement and density limit in stellarator/heliotron devices." Nuclear Fusion, vol. 30, no. 1, p. 11 (1990).

21 J. Miyazawa, R. Sakamoto, S. Masuzaki et al. "Density limit study focusing on the edge plasma parameters in LHD." Nuclear Fusion, vol. 48, no. 1, p. 015003 (2008).

${ }^{22}$ L. Giannone, R. Burhenn, K. McCormick et al. "Radiation power profiles and density limit with a divertor in the W7-AS stellarator." Plasma Physics and Controlled Fusion, vol. 44, no. 10, p. 2149 (2002).

23 A. Dinklage, M. Yokoyama, K. Tanaka et al. "Inter-machine validation study of neoclassical transport modelling in medium- to high-density stellaratorheliotron plasmas." Nuclear Fusion, vol. 53, p. 063022 (2013).

${ }^{24}$ C. D. Beidler, K. Allmaier, M. Y. Isaev et al. "Benchmarking of the monoenergetic transport coefficients - results from the International Collaboration on Neoclassical Transport in Stellarators (ICNTS)." Nuclear Fusion, vol. 51, p. 076001 (2011).

25 A. A. Subbotin, M. I. Mikhailov, V. D. Shafranov et al. "Integrated physics optimization of a quasi-isodynamic stellarator with poloidally closed contours of the magnetic field strength." Nuclear Fusion, vol. 46, p. 921 (2006).

${ }^{26}$ H. E. Mynick, N. Pomphrey and P. Xanthopoulos. "Optimizing Stellarators for Turbulent Transport." Physical Review Letters, vol. 105, p. 095004 (2010).

27 Y. Turkin, H. Maaßberg, C. D. Beidler et al. "Current Control by ECCD for W7-X." Fusion Science and Technology, vol. 50, p. 387 (2006).

${ }^{28}$ Y. Turkin, C. D. Beidler, H. Maaßberg et al. "Neoclassical transport simulations for stellarators." Physics of Plasmas, vol. 18, p. 022505 (2011).

${ }^{29}$ A. A. Galeev and R. Z. Sagdeev. "Theory of Neoclassical Diffusion." Reviews of Plasma Physics, vol. 7, p. 257 (1979).
${ }^{30}$ D. D.-M. Ho and R. M. Kulsrud. "Neoclassical transport in stellarators." Physics of Fluids, vol. 30, p. 442 (1987).

${ }^{31}$ W. I. van Rij and S. P. Hirshman. "Variational bounds for transport coefficients in threedimensional toroidal plasmas." Phyiscs of Fluids B: Physics of Plasmas, vol. 1, p. 563 (1989).

${ }^{32}$ S. P. Hirshman, K. C. Shaing, W. I. van Rij et al. "Plasma transport coefficients for nonsymmetric toroidal confinement systems." Physics of Fluids, vol. 29, p. 2951 (1986).

${ }^{33}$ K. C. Shaing. "Stability of the radial electric field in a nonaxisymmetric torus." Physics of Fluids, vol. 27, p. 1567 (1984).

${ }^{34}$ H. Maßßberg, R. Brakel, R. Burhenn et al. "Transport in stellarators." Plasma Physics and Controlled Fusion, vol. 35, p. B319 (1993).

${ }^{35}$ U. Stroth. "A comparative study of transport in stellarators and tokamaks." Plasma Physics and Controlled Fusion, vol. 40, p. 9 (1998).

${ }^{36}$ P. Xanthopoulos, F. Merz, T. Grler et al. "Nonlinear Gyrokinetic Simulations of Ion-Temperature-Gradient Turbulence for the Optimized Wendelstein 7-X Stellarator." Physical Review Letters, vol. 99, p. 035002 (2007).

${ }^{37}$ J. H. E. Proll, P. Xanthopoulos and P. Helander. "Collisionless microinstabilities in stellarators. II. Numerical simulations.” Physics of Plasmas, vol. 20, p. 122506 (2013).

${ }^{38}$ K. Lackner and N. A. O. Gottardi. "Tokamak confinement in relation to plateau scaling." Nuclear Fusion, vol. 30, p. 767 (1990). 